\title{
SUMMARIES OF ADDRESSES OF PRESIDENTS OF SECTIONS
}

\section{MATHEMATICAL NOTATION}

$I^{\mathbb{N}}$ $\mathrm{N}$ his presidential address to Section A (Mathematics and Physics), Prof. E. H. Neville discusses mathematical notation.

The great contribution of the nineteenth century to the subject was the acceptance of the non-numerical variable. Evidence of the belief that the variable must be in some sense numerical survives in the name of the complex number, and is to be found also in early writings on matrices and on quaternions. Cayley speaks of "the matrix considered as a single quantity", and it was not until the matrix was presented as an operator transforming one set of variables into another that it had the individuality which qualified it to be the element of an algebra. The same sort of individuality was conferred on the complex number by Argand and Wessel to end the scandal of 'the square root of minus one'. The adventurous calculus of operations of the nineteenth century was chopped to death by logic, but not before the enlarged conception of the variable was firmly established.

Notation is not right or wrong, but only good or bad. The most irritating of notational misfortunes are those where we should all like a slight modification in a usage that has become classical. No one can doubt that if we had a free hand to-day we should have a symbol for the radial measure of one right angle, not of two; the economy would be incalculable. William Jones is not to blame, nor even Euler, for the evidence is cumulative and was not in their possession; now it is too late to make a change. The case of the gamma function is more complicated. Gauss' $\Pi x$ is identical with Legendre's $\Gamma(1+x)$, and there is no doubt that Gauss' argu. ment is the more convenient : to quote Jeffreys, the 1 which Legendre took over from Euler is " $a$ minor but continual nuisance". We can agree to Gauss" form of the function but not to his appropriation of $I I$; the notation $x$ !, which is gaining currency, creates difficulties of its own.

If two symbols are equivalent it does not alwrys follow that one of them is redundant, and however closely two functions are related there may be sound reasons for retaining symbols for them both; a mathematical sense of proportion is often exhibited in the play made with alternatives. The theory of elliptic functions needs both the classical notation $K$, $i K^{\prime}$ and the structural notation $K_{c}, K_{n}$ for quarterperiods; we can seldom make the best of two worlds unless we can speak two languages. In the same theory, we cannot express satisfactorily the theorems which depend only on the period lattice and also the theorems which depend on the labelling of this lattice unless we have symbols both for the parameter $c$ and for the modulus $k$, although $c$ is only $k^{2}$. The most notorious illustration of this principle is the scalar product of two vectors; the physicist and the engineer need the product $a b \cos \varepsilon$; but it was sheer ignorance to suppose that Hamilton could have accepted this product rather than its negative as fundamental in his calculus.

The most far-reaching innovation in formal symbolism in the present century has been Einstein's summation convention, which reduces to manageable proportions the labour of applying the tensor calculus, and has effected great simplifications in the presentation of co-ordinate geometry, of vector algebra, and of the theory of determinants. This convention operates within the field of the traditional notation of mathematics. The really revolutionary change that has been taking place at the same time is the permeation of mathematics by the symbolism of logic. English mathematicians have learned from "Principia Mathematica" that this symbolism not only provides systematic abbreviations for phrases in perpetual use, but also compels a careful analysis of the assertions to be expressed and incorporates an inferential calculus which can lead to an immense economy of actual thinking; the notation for orders of magnitude carries out these same tasks in its own sphere.

While logical symbols are being used more and more in the older parts of mathematics, newer subjects are tending more and more to ideography. In topology, in group theory, and in algebra in the modern sense, theorems which would have been enunciated verbally in 1900 are now enunciated symbolically. The notation controls the emphasis, reduces a long-winded corollary to a brief formula, renders accessible regions which it would have been humanly impossible to explore with words alone. These developments belong to groups of specialists rather than to the whole community of mathematicians, but it is the freedom of the community from conventional restraints in the use of notation that has made it possible for the groups fully to satisfy their own needs.

\section{MODERN DEVELOPMENTS IN CARBOHYDRATE CHEMISTRY}

$T$

HE period between the two Birmingham meetings of the British Association in 1913 and in 1950 has been noteworthy for the surprising progress made in the chemistry of natural products. This has been specially marked in the carbohydrate group, and the main theme of Prof. E. L. Hirst's presidential address to Section $B$ (Chemistry) is the development of the ideas and methods which have rendered possible the present-day knowledge of the structure of the simple and complex sugars. This knowledge, so widely spread in industry, biology and medicine are the ramifications of carbohydrate chemistry, is of vital importance for the solution of problems in many other fields of inquiry. At the time of the Birmingham meeting in 1913, progress in the chemistry of the simple sugars was retarded by lack of precise knowledge of ring structures, and only the most meagre information was available concerning the disaccharides and the polysaccharides.

New methods of attack were necessary, and the requisite pioneer work, which enabled the unsubstituted reactive hydroxyl groups of sugars to be rendered chemically inert without alteration of ring structure, was carried out in the St. Andrews laboratories by Purdie and Irvine. Oxidation of the fully methylated methylglycosides was then investi- 
gated and developed into a general method for the determination of ring structures. By means of this technique and ingenious modifications of it, such as the oxidation of the fully methylated aldobionic acids in the disaccharide group, the workers of the Birmingham school, under the late Sir Norman Haworth, in a comprehensive series of investigations, determined the structures of the stable methyl glycosides (later termed pyranosides from the six-membered ring present in the molecule), the furanosides and the important naturally occurring disaccharides and trisaccharides such as cellobiose, maltose, sucrose, gentiobiose and raffinose. In many cases alternative methods, analytical and synthetical, afterwards provided confirmatory proof of the accuracy of the results obtained by the methylation method. One of the most versatile of the newer techniques is oxidation by periodic acid, introduced by Malaprade for the oxidative degradation of glycols. Besides numerous important applications in the mono- and oligo-saccharide groups, this reagent is of special value in the study of the complex polysaccharides; for it can be employed to gain quantitative evidence of the presence of end-groups, and, by examination of the hydrolysis products obtained from the oxidized polysaccharide, information can be obtained concerning the mode of linkage of individual residues in the polymer.

Another recent development of the greatest importance to carbohydrate chemistry is the use of chromatography for the quantitative separation of mixtures of sugars, whether unsubstituted or in the form of their methyl ethers. So delicate and accurate has this technique become that for the time being a limiting factor in the study of the complex carbo. hydrates resides not so much in the separation and classification of the various sugar residues as in the difficulty encountered in hydrolysing the polysaccharides without damage to the component sugars. Besides applying these methods to the undegraded polysaccharides, present-day investigators attempt to simplify their problems by examining the structure of degraded fragments obtained by partial hydrolysis.

In those ways, aided also by highly specialized physico-chemical techniques such as X-ray analysis and sedimentation in the ultracentrifuge, sufficient information may be gained on which to base the general structure of a polysaccharide. In some cases, for example, cellulose, which consists essentially of long chains of $\beta-1,4$-linked glucose residues, the structure is known in some detail; but even here there remains a fine structure of great importance to the industrial chemist about which little is yet known. The main structure of the amylose component of starch ( $x$-1,4-linked glucose residues) is also known with some certainty. Much still remains to be learned concerning the amylopectin component of starch with its many branched chains and five per cent of its glucose residues present as end-groups.

Reference has been made to the special problems presented by the xylans, arabans, pectins and alginic acid, and it has been pointed out that, difficult as all these problems are, they are simple in the sense that each polysaccharide contains only one kind of sugar residue. The plant gums and mucilages and many polysaccharides of importance in chemotherapy present much more complex problems in that they contain a variety of sugar residues, and it is therefore necessary to ascertain the order in which these are united together in the molecule. Only a beginning has yet been made with structural studies on these groups, and Prof. Hirst concludes with a review of the position now reached, with special reference to the chemistry of gum arabic.

\section{STONY METEORITES}

$\mathrm{D}$ R. W. CAMPBELL SMITH, in his presidential address to Section C (Geology), points out that among the thirteen hundred or so known meteorites recorded in the past hundred and fifty years, some seven hundred and sixty are 'stony meteorites' consisting mainly of ferromagnesian silicates. The elements found in meteorites do not differ from those known on the earth, and the silicate minerals of the stony meteorites are varieties of the same species as those found in basic and ultrabasic igneous rocks.

About ninety per cent of all stony meteorites contain varying proportions of nickel-iron, ranging from 4 to 25 per cent, and these (with half a dozen exceptions) are characterized also by the presence of small, spherical bodies ('chondrules') consisting of pyroxene or olivine, or both together. These meteorites are called chondrites. Their chondritic structure and nickel-iron content make them quite unlike any known terrestrial rocks. The remaining ten per cent of stony meteorites, containing no chondrules, are called 'achondrites'. Some of these show a partial resemblance in mineral composition and texture to igneous rocks. By applying to them the methods used in the study of igneous rocks and rockforming minerals, we may hope to obtain some insight into the conditions under which meteorites were first formed and what changes they have since suffered.

One group of the achondrites, the eucrites and howardites, represented by about twenty-five falls, comprise meteorites of which the constituent minerals are feldspar (bytownite-anorthite) and pyroxene (mainly pigeonite). They are, with one exception, intensely brecciated; but they contain many frag. ments which resemble in texture some ophitic dolerites and basalts, and these are regarded as products of direct crystallization from molten magma.

The other achondrites contain little or no feldspar, consist of magnesium-iron-pyroxenes and olivine, and, except when brecciated, present a holocrystalline granular texture. Their mineral assemblage falls aimost wholly within the $\mathrm{MgO}-\mathrm{FeO}-\mathrm{SiO}_{2}$ system fully investigated experimentally by $\mathrm{N}$. L. Bowen and J. F. Schairer. The conditions of crystallization of the several phases in this system show that one cannot explain the crystalline granular achondrites as the result of direct crystallization from a magma of their own bulk composition. They must be regarded rather as the result of crystal-settling in a differentiating magma. Such a process requires not only bodies of magma of considerable size but also a gravity field sufficient to make settling of erystals in a magma possible. Both requisites imply that the material of these achondritic meteorites formed within a body comparable in size with our earth.

A similar settling of magnesium silicate minerals, but mixed up in some way with nickel-iron, may account for the original materials of the chondritic meteorites; but their subsequent changes in com. 